\title{
Levadura viva (Saccharomyces cerevisiae) en la degradabilidad in vitro de una dieta para bovinos basada en forraje más concentrado
}

\section{Live Yeast (Saccharomyces cerevisiae) in the in vitro Degradability of a Diet for Cattle}

Piedad Francisca Yépez Macías ${ }^{1,2},{ }^{\bullet}$ Juan Avellaneda-Cevallos ${ }^{2,3}$, María de Fátima Villegas Jurado ${ }^{4}$, Víctor Hugo Godoy Espinoza ${ }^{1}$, Néstor Vicente Acosta Lozano ${ }^{5}$, Carlos Alberto Molina Hidrovo ${ }^{3}$

${ }^{1}$ Universidad Técnica Estatal de Quevedo. Facultad de Ciencias Pecuarias. Carrera de Ingeniería Zootécnica. Campus Finca Experimental "La María”. CP. 121250.Km. 7 1⁄2 vía El Empalme, cantón Mocache, Los Ríos. Ecuador. pyepez@uteq.edu.ec ${ }^{2}$ Universidad Tecnológica Equinoccial. Facultad de Ciencias de la Ingeniería e Industrias. Unidad de Posgrado. CP. 230102. Via Chone, Km 41⁄2. Cantón Santo Domingo de los Colorados, Santo Domingo de los Tsáchilas. Ecuador. ${ }^{3}$ Instituto Nacional de Investigaciones Agropecuarias-INIAP. Estación Experimental Tropical Pichilingue. CP. 121250.Km 5 1/2 vía Quevedo-El Empalme, cantón Mocache,Los Ríos. Ecuador. ¡juan.avellaneda@gmail.com; carlos.molina@iniap.gob.ec

${ }^{4}$ Universidad Agraria del Ecuador campus Milagro. CP. 070353. Avenida Jacobo Bucaram y Emilio Mogner, cantón Milagro, Guayas. Ecuador. fvillegas@uagraria.edu.ec

${ }^{5}$ Universidad Estatal Península de Santa Elena. Facultad de Ciencias Agrarias. Carrera de Ingeniería Agropecuaria. CP. 240350. Km. 1 vía La Libertad-Santa Elena, cantón La Libertad, Santa Elena.Ecuador. acoslones@yahoo.es

Rec.: 18.10.2016. Acept.: 20.01.2017. Publicado el 1 de junio de 2017

Resumen

Se evaluaron cuatro niveles de cultivo de levadura viva (Saccharomyces cerevisiae) en la digestibilidad in vitro de una dieta para bovinos basada en forraje más concentrado. Se utilizó líquido ruminal de dos toros fistulados, como inoculo bacteriano. Se emplearon dos incubadores Ankom ${ }^{\circledR}$ para digestibilidad in vitro, donde se depositaron bolsas F57 (Ankom Technology) que contenían las muestras de tratamientos experimentales. Los tratamientos fueron; T1: dieta testigo a base de forraje más concentrado, T2: T1 más $1 \mathrm{~g}$ de CL (Cultivo de levadura) $\mathrm{kg}^{-1}$ de alimento, T3: T1+2 $\mathrm{g}$ de CL kg${ }^{-1}$ de alimento y T4: T1+3 g de CL kg-1 de alimento. La incubación se efectuó en siete tiempos; y la degradación se detuvo mediante refrigeración a $4^{\circ} \mathrm{C}$. El experimento duró 90 días. Se evidenció a las 3,12 y 72 h de incubación ruminal, la degradabilidad in vitro (DRIV) de la materia seca (DIMS), no presentó cambios por efecto de la suplementación del CL. No obstante, aunque de una manera no lineal, este CL provocó diferencias para los periodos de incubación de $0,6,24$ y 48 horas. La DRIV de la materia orgánica (DIMO) tampoco presentó un comportamiento lineal por efecto de la adición creciente del CL a la dieta base, existiendo diferencias sólo en los tiempos de incubación de 3, 24 y 48 horas, pero no en el mayor tiempo de incubación (72 h). Los CL no provocan cambios claros en la degradabilidad de las dietas estudiadas.

Palabras clave: cultivo de levadura, incubación in vitro, king grass, líquido ruminal
Gour levels of live yeast (Saccharomyces cerevisiae) $\boldsymbol{\Gamma}$ culture in the in vitro digestibility of a more concentrated forage based cattle diet were evaluated. Ruminal fluid of two fistulated bulls was used, as a bacterial inoculum. Two Ankom ${ }^{\circledR}$ incubators were used for in vitro digestibility, where F57 bags (Ankom Technology) containing the samples of experimental treatments were deposited. The treatments were; T1: control diet with more concentrated forage, T2: T1 plus 1 $\mathrm{g}$ of CL (Yeast Culture) $\mathrm{kg}^{-1}$ of feed, T3: T1 plus $2 \mathrm{~g}$ of CL kg-1 of feed and T4: T1 plus $3 \mathrm{~g}$ of CL kg${ }^{-1}$ of food. The experiment was developed in the Laboratory of Nutrition Rumiology and Metabolism with a duration of 90 days. In vitro dry matter degradability (DIMS) showed no changes at 3,12 and $72 \mathrm{~h}$ of ruminal incubation because of CL supplementation. However, although in a non-linear manner, this CL caused differences for the incubation periods of $0,6,24$ and 48 hours. The DRIV of organic matter (DIMO) did not present a linear behavior because of the increasing addition of CL to the base diet. There were differences only in incubation times of 3,24 and 48 hours, but not in the longest incubation time ( $72 \mathrm{~h})$. CLs do not cause clear changes in the degradability of the diets studied.

Key words: yeast culture, in vitro incubation, king grass, ruminal liquid 


\section{Introducción}

$\mathrm{L}_{\mathrm{s}}^{\mathrm{o}}$ os rumiantes poseen la capacidad de utilizar los forrajes y alimentos toscos, los cuales son poco utilizados por otras especies domésticas. Esto ocurre en el rumen gracias a la presencia de microorganismos que mediante procesos de fermentación degradan la dieta consumida generando ácidos grasos volátiles (AGV), que son la principal fuente de energía para el rumiante y los propios microorganismos ruminales. Además, estas bacterias y protozoarios aportan una cantidad muy importante de la proteína que necesitan los animales día a día (Davis, 1993); sin embargo en los últimos tiempos, los rumiantes están siendo sometidos a dietas con alto niveles de concentrados con la finalidad de incrementar su nivel productivo, causando estos nuevos sistemas, algunos problemas metabólicos a los que hay que darle solución.

Una de esas alternativas, para minimizar el impacto ruminal de las dietas que contienen granos, es el uso de levaduras vivas ya que tienen muchas ventajas (Evans et al., 2012), mismas que además de contribuir a mejorar la eficiencia alimenticia (White et al., 2008; Holtshausen y Beauchemin, 2010; Ondarza et al., 2010a), producción y contenido de proteina en leche (Shaver y Garrett, 1997; Ondarza et al., 2010b), ganancia diaria de peso (Ponce et al., 2012), incremento de las poblaciones de microorganismos totales del rumen (Fokkink et al., 2009) y en muchos casos el incremento en la digestibilidad de los forrajes (Bacha, 2002), aunque en otros no presentan efecto (Hinman et al., 1998; O'Connor et al., 2002). Los betaglucanos presentes en la pared externa de las levaduras estimula el sistema de defensa natural del organismo; además de estimular de forma selectiva el crecimiento de las poblaciones de bacterias consumidoras de lactato (Megaspharera elsdenii y Selenomonas ruminantium) lo que reduce la presencia de ácido láctico, evitando así las caídas pronunciadas de $\mathrm{pH}$ ruminal, lo que disminuye la incidencia de acidosis y por lo tanto los problemas digestivos, las cojeras y los altos conteos de células somáticas asociadas a esta causa (Clayton et al., 1999; Bacha, 2002).

Por ello, se buscó estudiar los cambios que experimentan en términos de degradabilidad in vitro, dietas que se basan en forraje+concentrado, a las cuales se les adiciona levaduras vivas como mejoradoras de las características de uso ruminal de los componentes nutricionales.

\section{Materiales y métodos}

$\mathrm{E}_{\mathrm{R}}^{\mathrm{st}}$ ste estudio se llevó a cabo en el Laboratorio de Rumiología y Metabolismo Nutricional, situado en el Campus experimental "La María" de la Universidad
Técnica Estatal de Quevedo, Facultad de Ciencias Pecuarias, ubicada en el km. 7 vía Quevedo-El Empalme, provincia de Los Ríos. La ubicación geográfica es de $01^{\circ}$ $0.6^{\circ}$ de latitud sur, $79^{\circ} 29^{\circ}$ de latitud oeste, a una altura de $73 \mathrm{msnm}$, condiciones climáticas bosque humedo Tropical (bh-T), con precipitación promedio mensual $140.70 \mathrm{~mm}$, temperatura promedio $25.80{ }^{\circ} \mathrm{C}$, Heliofanía 97.53 horas luz y humedad relativa $89.40 \%$ (INAMHI, 2015). El estudio tuvo una duración de tres meses. Se utilizó un diseño completo al azar. Se empleó un total de cuatro tratamientos con seis repeticiones, siendo cada bolsa de Ankom ${ }^{\circledR}$ F57 una repetición (168 bolsas de Ankom ${ }^{\circledR}$ F57: 4 tratamientos $\mathrm{x} 6$ repeticiones y 7 tiempos de incubación ruminal). Cada tratamiento representó la dieta base (Cuadro 1) a la que se le adicionó los diferentes niveles de levaduras $(0,1,2$ y 3 $\mathrm{g} \mathrm{kg}^{-1}$ ). Para el procesamiento de la información se utilizó el software estadistico INFOSTAT versión 2008 (Di Rienzo et al., 2008), y para establecer la diferencia de medias se empleó la prueba de Tukey $(\mathrm{p} \leq 0.05)$.

Manejo del experimento. El líquido ruminal fue recolectado de dos toros fistulados de raza Brahman con un peso aproximado de $450 \pm 20 \mathrm{~kg}$ que fueron alimentados con pasto saboya, agua y minerales ad libitum. El líquido ruminal se diluyó (4:1) con saliva artificial de $\mathrm{pH} 6.8$ (Tilley y Terry, 1963). El pasto king grass fresco fue cortado a una edad de 75 días, secado a la intemperie y molido a un tamaño de $1 \mathrm{~mm}$. En el alimento (pasto más concentrado), se determinó materia seca (MS) y orgánica (MO); proteína total (PT; $\mathrm{N}$ x 6.25) y cenizas o materia inorgánica (C o MI) (AOAC, 1990). Para la digestibilidad in vitro (DIV) de la MS (DIVMS), MO (DIVMO) y de las C (DIVC) se utilizó la primera fase de la técnica de Tilley y Terry (1963), mezclando saliva artificial (McDougall, 1948) y líquido ruminal (relación 4:1). El medio (saliva de McDougall más líquido ruminal) se depositó en los frascos de anaerobiosis de $2000 \mathrm{~mL}$ de un equipo Ankom ${ }^{\circledR}$ Technology; así como, las bolsas F57 con $500 \mathrm{mg}$ de cada tratamiento previamente molido con malla de $1 \mathrm{~mm}$; este medio fue gaseado con $\mathrm{CO}_{2} \mathrm{y}$ tapados para mezclar su contenido; la incubación se efectuó durante $0,3,6,12,24,48$ y 72 horas; y la degradación se detuvo mediante refrigeración a $4{ }^{\circ} \mathrm{C}$.

\section{Resultados y discusión}

\section{Degradabilidad in vitro de la materia seca}

Los resultados experimentales (Cuadro 2), demuestran diferencias a las 0, 6, 24 y 48 horas de incubación ruminal, la degradabilidad in vitro de la materia seca (DIMS) presentó cambios $(\mathrm{p}<0.05)$ por efecto de la suplementación del cultivo de levadura (CL). Aunque de una manera no lineal, fue difícil de explicar, pues no existieron indicios claros para poder 
Cuadro 1. Composición porcentual de ingredientes y química proximal de las dietas experimentales

\begin{tabular}{lr}
\hline Ingrediente & Composición (\%) \\
\hline Pasto king grass & 60.00 \\
Maíz & 16.78 \\
Polvillo de arroz & 7.20 \\
Torta de soya & 12.00 \\
Aceite vegetal & 2.09 \\
Carbonato de calcio & 0.80 \\
Fosfato monocálcico & 0.53 \\
Sal & 0.60 \\
\hline Total & 100 \\
\hline Nutriente (en base seca) & 2858 \\
\hline Energía metabolizable, $\mathrm{Kcal} \mathrm{kg}^{-1}$ & 12.06 \\
Proteína cruda total & 3.05 \\
Grasa cruda total & 15.37 \\
Fibra cruda total & 0.69 \\
Calcio & 0.34 \\
Fósforo disponible & 0.83 \\
Cloro & Composición química (\%) \\
\hline
\end{tabular}

Fuente: Laboratorio de Rumiologia y Metabolismo Nutricional y Laboratorio de Bromatología de la Universidad Técnica Estatal de Quevedo

Cuadro 2. Efecto tres niveles de una levadura viva constituida por Saccharomyces cerevisiae sobre la degradabilidad de la materia seca (\%) de una dieta para bovinos basada en forraje más concentrado

\begin{tabular}{cccccc}
\hline $\begin{array}{c}\text { Tiempo de } \\
\text { incubación } \\
\text { ruminal }\end{array}$ & \multicolumn{4}{c}{ Tratamiento experimental } & \multirow{2}{*}{$\boldsymbol{P}<$} \\
\cline { 2 - 5 } & $\mathbf{T 1}$ & $\mathbf{T} 2$ & $\mathbf{T 3}$ & $\mathbf{T 4}$ & \\
\hline 0 & $17.28 \mathrm{a}$ & $14.79 \mathrm{~b}$ & $17.39 \mathrm{a}$ & $18.14 \mathrm{a}$ & 0.002 \\
3 & $20.63 \mathrm{a}$ & $20.42 \mathrm{a}$ & $21.14 \mathrm{a}$ & $19.80 \mathrm{a}$ & 0.465 \\
6 & $24.35 \mathrm{a}$ & $21.53 \mathrm{~b}$ & $23.74 \mathrm{a}$ & $23.21 \mathrm{ab}$ & 0.006 \\
12 & $34.22 \mathrm{a}$ & $30.95 \mathrm{a}$ & $32.89 \mathrm{a}$ & $31.57 \mathrm{a}$ & 0.139 \\
24 & $43.03 \mathrm{~b}$ & $44.23 \mathrm{ab}$ & $44.97 \mathrm{ab}$ & $45.72 \mathrm{a}$ & 0.038 \\
48 & $57.67 \mathrm{a}$ & $52.68 \mathrm{~b}$ & $56.50 \mathrm{a}$ & $57.45 \mathrm{a}$ & 0.003 \\
72 & $64.49 \mathrm{a}$ & $63.24 \mathrm{a}$ & $64.90 \mathrm{a}$ & $63.11 \mathrm{a}$ & 0.203 \\
\hline
\end{tabular}

Medias con una letra común, no son significativamente diferentes (Tukey, $\mathrm{p}>0.05$ ). T1: Testigo, sin cultivo de levadura $\mathrm{kg}^{-1}$ de alimento; $\mathrm{T} 2: \mathrm{T} 1+1 \mathrm{~g}$ de cultivo de levadura $\mathrm{kg}^{-1}$ de alimento; $\mathrm{T} 3: \mathrm{T} 1+2 \mathrm{~g}$ de cultivo de levadura $\mathrm{kg}^{-1}$ de alimento; $\mathrm{T} 4: \mathrm{T} 1+3 \mathrm{~g}$ de cultivo de levadura $\mathrm{kg}^{-1}$ de alimento.

determinar esta tendencia, se puede presumir que en el nivel intermedio de adición del CL, hay un perfil ruminal favorable.

Sin embargo, estos resultados, fueron diferentes a los reportados por Boas et al. (2007), quienes evaluaron la adición de tres cepas de levadura Saccharomyces cerevisiae contra un tratamiento testigo, sobre la degradabilidad ruminal in situ de la materia seca de la caña de azúcar. Esta discrepancia, pudo deberse a que estos autores midieron el efecto de tres diferentes cepas de levaduras, en lugar de un nivel incremental del mismo producto, estos efectos positivos del CL sustentan una de las bondades de estas, más aún, porque la fuente alimenticia investigada fue estrictamente forrajera. 
Lo anteriormente indicado es probable, por las peculiaridades que posee $S$. cerevisiae ya que uno de sus mecanismos de acción es la eliminación del oxígeno del ambiente ruminal, lo que promueve que las bacterias anaeróbicas estrictas como las celulolíticas tengan un mejor crecimiento (Mcleod et al., 1991); propendiendo al uso ruminal de la pared celular de los forrajes por mecanismos degradativos (Galloway et al., 1991). Por otra parte, Gomes et al. (2010), reportaron la no existencia de diferencias en la degradabilidad ruminal in situ de la MS de una dieta testigo alta en concentrado con bagazo de caña como fuente forrajera más un cultivo de levaduras vivas (CLV) de Saccharomyces cerevisiae (Beef

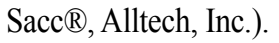

\section{Degradabilidad in vitro de la materia orgánica}

La degradabilidad ruminal in vitro de la materia orgánica (DIMO; Cuadro 3), tampoco presentó un comportamiento lineal por efecto de la adición creciente del CL a la dieta base, existiendo diferencias $(\mathrm{p}<0.05)$ sólo en los tiempos de incubación de 3 , 24 y 48 horas; estos resultados concuerdan con los reportados por Tripathi y Karim (2010) quienes evaluaron el efecto de la alimentación de CL en la mejora de la utilización de nutrientes de una dieta de concentrados mezclado con $25 \%$ de forraje, sosteniendo que la carencia de efecto puede deberse al alto nivel de concentrado de la dieta.

Cuadro 3. Efecto de tres niveles de una levadura viva constituida por Saccharomyces cerevisiae sobre la degradabilidad de la materia orgánica (\%) de una dieta para bovinos basada en forraje más concentrado

\begin{tabular}{cccccc}
\hline $\begin{array}{c}\text { Tiempo de } \\
\text { incubación } \\
\text { ruminal }\end{array}$ & $\mathbf{T 1}$ & $\mathbf{T 2}$ & $\mathbf{T 3}$ & $\mathbf{T 4}$ & P $<$ \\
\cline { 2 - 5 } & $13.74 \mathrm{a}$ & $13.25 \mathrm{a}$ & $12.44 \mathrm{a}$ & $14.00 \mathrm{a}$ & 0.260 \\
3 & $17.48 \mathrm{ab}$ & $18.71 \mathrm{a}$ & $16.25 \mathrm{ab}$ & $16.10 \mathrm{~b}$ & 0.027 \\
6 & $20.02 \mathrm{a}$ & $19.74 \mathrm{a}$ & $18.26 \mathrm{a}$ & $20.47 \mathrm{a}$ & 0.102 \\
12 & $32.60 \mathrm{a}$ & $31.24 \mathrm{a}$ & $29.25 \mathrm{a}$ & $29.55 \mathrm{a}$ & 0.095 \\
24 & $41.91 \mathrm{c}$ & $44.26 \mathrm{ab}$ & $43.50 \mathrm{bc}$ & $45.82 \mathrm{a}$ & 0.001 \\
48 & $58.78 \mathrm{a}$ & $54.67 \mathrm{c}$ & $55.80 \mathrm{bc}$ & $58.46 \mathrm{ab}$ & 0.001 \\
72 & $65.70 \mathrm{a}$ & $64.11 \mathrm{a}$ & $64.69 \mathrm{a}$ & $64.70 \mathrm{a}$ & 0.383 \\
\hline
\end{tabular}

Medias con una letra común, no son significativamente diferentes (Tukey, $\mathrm{p}>0.05$ )

El aumento de la digestibilidad del forraje de pobre calidad con frecuencia se reporta como respuesta a la suplementación de levaduras vivas (Guedes et al., 2008) misma que puede ser debida a la inducción del crecimiento de las bacterias celulolíticas (Plata et al.,
1994); sin embargo, en la presente investigación los animales fueron alimentados con una dieta con $40 \%$ de concentrado; esta condición pudiera haber limitado la presencia de resultados positivos sobre la DIMO; tal como lo reportó Tripathi y Karim (2010).

Cuadro 4. Efecto tres niveles de una levadura viva constituida por Saccharomyces cerevisiae sobre la biodisponibilidad de la materia inorgánica (\%) de una dieta para bovinos basada en forraje más concentrado

\begin{tabular}{cccccc}
\hline $\begin{array}{c}\text { Tiempo de } \\
\text { incubación } \\
\text { ruminal }\end{array}$ & T1 & T2 & T3 & T4 & \multirow{2}{*}{ Tratamiento experimental } \\
\cline { 2 - 5 } & $40.23 \mathrm{a}$ & $27.48 \mathrm{~b}$ & $47.93 \mathrm{a}$ & $49.09 \mathrm{a}$ & 0.0002 \\
\hline 0 & $41.01 \mathrm{ab}$ & $34.58 \mathrm{~b}$ & $51.25 \mathrm{a}$ & $47.73 \mathrm{a}$ & 0.0031 \\
3 & $52.32 \mathrm{ab}$ & $36.33 \mathrm{c}$ & $57.53 \mathrm{a}$ & $44.60 \mathrm{bc}$ & 0.0002 \\
6 & $44.69 \mathrm{ab}$ & $28.54 \mathrm{~b}$ & $55.26 \mathrm{a}$ & $47.73 \mathrm{ab}$ & 0.0104 \\
12 & $50.24 \mathrm{a}$ & $44.03 \mathrm{a}$ & $53.93 \mathrm{a}$ & $47.05 \mathrm{a}$ & 0.3106 \\
24 & $50.52 \mathrm{a}$ & $36.33 \mathrm{~b}$ & $60.68 \mathrm{a}$ & $52.19 \mathrm{a}$ & 0.0008 \\
48 & $56.73 \mathrm{ab}$ & $56.02 \mathrm{ab}$ & $66.12 \mathrm{a}$ & $53.77 \mathrm{~b}$ & 0.0255 \\
72 & &
\end{tabular}

Medias con una letra común, no son significativamente diferentes (Tukey, $\mathrm{p}>0.05$ ) 
Biodisponibilidad in vitro de la materia inorgánica

La biodisponibilidad de MI in vitro presentó un alto grado de variabilidad e inconsistencia en la respuesta, en función de los tiempos y grado de degradabilidad ruminal, motivo por el cual no se pudo de manera concluyente definir la presencia de algún efecto negativo o positivo del CL, en las dietas en estudio. Sin embargo, existió la presencia de diferencias $(\mathrm{p}<0.05)$ en casi todos los tiempos de incubación evaluados (Cuadro 4).

\section{Conclusiones}

$\mathrm{L}_{\mathrm{y}}^{\mathrm{as}}$ as dietas basadas en forrajes más concentrado mejoradoras de los componentes nutricionales, presentó efectos en la DIVMS, DIVMO y DIVMI con variabilidad, acción que no permite concluir concretamente sobre el efecto del cultivo de levadura.

\section{Bibliografía}

AOAC. 1990. Official Methods of Analysis. Washington D.C.: Of the Association of Official Analytical Chemists.

Bacha, A. (2002). Trastornos ruminales en el vacuno lechero: Un enfoque práctico. XVIII. Curso de Especialización Avances en Nutrición y Alimentación Animal. FEDNA. México

Bôas, W., Franzolin, R., \& Souza, N. (2007). Effect of addition of different strains of yeast (Saccharomyces cerevisiae) on liquid phase outflow rate, ruminal volume and in situ degradability in buffaloes fed on the sugar cane basis. Italian Journal of Animal Science, 6, 536539.

Clayton, EH., Lean, IJ., Rowe, JB., y Cox, JW. (1999). Effects of feeding virginiamycin and sodium bicarbonate to grazing lactating dairy cows. J Dairy Sci. 82(7):1545-54.

Davis, CL. (1993). Alimentación de la Vaca Lechera Alta Productora. Illinois, USA: University of Illinois.

Di Rienzo, JA., Casanoves, F., Balzarini, M., Gonzalez, L., Tablada, M., \& Robledo, C. (2008). InfoStat, versión 2008. (U. N. Córdoba, Ed.) Cordoba, Argentina: Grupo InfoStat.

Evans, E., Patterson, RJ., \& Clark, N. (2012). Effects of a supplemental enhanced yeast product on digestion and milk production in dairy cows. The Professional Animal Scientist, 28, 682-688.
Fokkink, WB., Hill, TM., Aldrich, JM., Bateman, H. G., \& Schlotterbeck, R. L. (2009). Effect of Yeast Culture, Fatty Acids, Whey, and a Peptide Source on Dairy Calf Performance. The Professional Animal Scientist, 25, 794-800.

Galloway, DL., Goetsch, AL., Sun, W., \& Forester, LA. (1991). Effect of addition of sodium bicarbonate salt, Aspergillus oryzae culture extract, niacin, lysine or phenylalanine to ground corn-based supplements on feed intake and digestion by Holstein steers consuming Bermuda grass (Cynodon dactylon) hay. Animal Feed Science and Technology, 32, 261-273.

Gomes, R., Antunes, M., Nogueira, J., Ítavo, C., \& Leme, P. (2010). Levaduras vivas e monensina em dietas de alto concentrado para bovinos: Parâmetros ruminais e degradabilidade "in situ". Revista Brasileira da Saúde e Produao Animail, 11(1), 202-216.

Guedes, CM., Goncalves, D., Rodríguez, A., \& Silva da, A. (2008). Effects of yeast Saccharomyces cerevisiae on ruminal fermentation and fiber degradation of maize silage in cows. Animal Feed Science and Technology, 145, 27.

Hinman, D., Sorensen, SJ., \& Momont, PA. (1998). Effect of Yeast Culture on Steer Performance, Apparent Diet Digestibility, and Carcass Measurements When Used in a Barley and Potato Finishing Diet. TYehaes tP Croufletusrsei oinn aBla Arlneyim anadl SPcoiteantot iFsti, 14, 173177.

INAMHI (Instituto Nacional de Metereología e Hidrología). (2015). Anuario de Metereología e Hidrologías. Estación Experimental Tropical Pichilingue. Instituto Nacional de Investigaciones Agropecuarias

Holtshausen, L., \& Beauchemin, KA. (2010). Supplementing Barley-Based Dairy Cow Diets with Saccharomyces cerevisiae. 26, 285-289.

McDougall, E. (1948). Studies on ruminant saliva. The composition and output of sheep's saliva. Biochemical Journal, 70, 99-109.

Mcleod, KR., Karry, KJ., Dawson, KA., \& Mitchell, GE. (1991). Influence of yeast culture and monensin on ruminal metabolic end products and feedlot performance. En T. P. Lyons, Biotechnology in the feed Industry. Nicholasville, Kentucky, USA: Alltech's Techical Publications.

O'Connor, MH., Martin, SA., \& Hill, GM. (2002). Effects of Saccharomyces cerevisiae in vitro Mixed Ruminal Microorganism Fermentation. The Professional Animal Scientist, 18, 358-362.

Ondarza, MB., Sniffen, CJ., Graham, H., \& Wilcock, P. (2010a). Effect of Supplemental Live Yeast on 
Yield of Milk and Milk Components in HighProducing Multiparous Holstein Cows. The Professional Animal Scientist, 26, 443-449.

Ondarza, MB., Sniffen, CJ., Dussert, L., Chevaux, E., Sullivan, J., \& Walker, N. (2010b). MultipleStudy Analysis of the Effect of Live Yeast on Milk Yield, Milk Component Content and Yield, and Feed Efficiency. The Professional Animal Scientist, 26, 661-666.

Plata, P., Mendoza, G., Barcena, R., \& Gonzalez, S. (1994). Effect of yeast culture (Saccheromyces cerevisiae) on neutral detergent fiber digestion in steer fed oat straw based diets. Animal Feed Science and Tecnology, 49, 203-210.

Ponce, CH., Schutz, JS., Elrod, CC., Anele, UY., \& Galyean, ML. (2012). Effects of dietary supplementation of a yeast product on performance and morbidity of newly received beef heifers. The Professional Animal Scientist, 28, 618-622.
Shaver, RD., \& Garrett, JE. (1997). Effect of Dietary Yeast Culture on Milk Yield, Composition, and Component Yields at Commercial Dairies. The Professional Animal Scientist, 13, 204-207.

Tilley, J., \& Terry, R. (1963). A two stage technique for the in vitro digestion of forage crops. Journas Brithish Grassland Society, 28, 104-111.

Tripathi, MK., \& Karim, SA. (2010). Effect of individual and mixed live yeast culture feeding on growth performance, nutrient utilization and microbial crude protein synthesis in lambs. Animal Feed Science and Technology, 155, 163171.

White, RA., Harrison, JH., Yoon, I., Sanchez, WK., \& Nicholson, N. (2008). Effect of Yeast Culture on Efficiency of Nutrient Utilization for Milk Production and Impact on Fiber Digestibility and Fecal Particle Size. The Professional Animal Scientist, 24, 114-119. 